\title{
Numerical Scattering Analysis for Two-Dimensional Dense Random Media: Characterization of Effective Permittivity
}

\author{
Kamal Sarabandi, Senior Member, IEEE, and Paul R. Siqueira
}

\begin{abstract}
In this paper, a new numerical method for determining effective permittivity of dense random media in two dimensions is presented. The core of the method is to compare the average scattered field of a random collection of scatterers confined within an imaginary boundary with the scattered field from a homogeneous dielectric of the same shape as the imaginary boundary. The two-dimensional (2-D) problem is aggressively studied here to provide insight into the dependence of the method's convergence on particle size, boundary shape, and boundary dimension. A novel inverse scattering method is introduced based on the method of moments (MoM), which greatly reduces the computation time and increases the flexibility of the procedure to analyze a variety of geometries. Results from this 2-D method may be used directly to compare with theoretical methods for determining effective permittivity such as the Polder-Van Santen mixing formula or field techniques such as the quasi-crystalline approximation.
\end{abstract}

Index Terms-Electromagnetic scattering, random media.

\section{INTRODUCTION}

A fundamental electromagnetic characteristic of a radar target is its permittivity or, equivalently, its propagation constant for a nonmagnetic material. Most natural targets at certain scales can be considered to be inhomogeneous, consisting of discrete components (or inhomogeneities) that are separate from a uniform background material. A uniform layer of snow for instance at low frequencies may appear to be a homogeneous medium, variations of interest being differences in snow depth or changes in the snow wetness on a scale similar to the observing wavelength. As the wavelength decreases there will come a point where it is necessary to consider the interaction of electromagnetic fields with individual crystals of ice that make up the snow layer. In such a case, the background medium may be considered the host and the ice crystals the inclusions. In both of these cases, it is of interest to theoretically predict the effective permittivity of the random medium. At low frequencies this is accomplished through the use of a mixing formula that directly models the interaction of electromagnetic fields with individual scattering particles through the polarizability tensor of isolated particles.

The most commonly used mixing formula is given by Polder and Van Santen [4]. The Polder-Van Santen mixing

Manuscript received October 24, 1995; revised August 14, 1996.

The authors are with the Radiation Laboratory, Electrical Engineering and Computer Science Department, University of Michigan, Ann Arbor, MI 48109 USA.

Publisher Item Identifier S 0018-926X(97)03205-5. formula relates the combined, effective dielectric constant of a collection of particles, to that of its constituents. The Polder-Van Santen model, as with many mixing formulas, relies on the observing wavelength to be much larger than the scale of the inhomogeneities within the material under study.

Approaches where the field interaction with the individual discontinuities are taken into account are termed field models, popular forms of which are Foldy's approximation, strong fluctuation theory, the quasi-crystalline approximation (QCA) and the quasi-crystalline approximation with coherent potential (QCA-CP) [1], [13]. With the exception of Foldy's approximation, these theories include additional interaction terms that occur because of multiple scattering between individual particles. These theories are thereby considered to be more accurate than mixing formulas when scattering from individual particles becomes a factor. Theoretical field approaches, however, must truncate this interaction at some given order to make the theory tractable - a truncation through which an approximation is introduced. The validity of this approximation for different situations is often difficult to assess, particularly when the variety of situations that need to be considered is large.

Validation of the mixing formula and field models is commonly accomplished through a comparison with physical experiment. Several drawbacks of experimental methods are that it is difficult to reduce natural variabilities in the sample under study and often it is not possible to gather sufficient and accurate ground truth to fully characterize the experiment. One such example is with the implementation of the quasi-crystalline approximation where knowledge of the pairdistribution function of particle positions is required. The pair-distribution function of particle positions is a parameter that is nearly impossible to measure for a naturally occurring random medium. These variabilities can be reduced by performing a controlled experiment in the lab, as in one notable experiment performed by Mandt [3] where individual glass spheres were placed at computer generated positions in Styrofoam. While experiments such as the one described here are an important component in analyzing the validity of field models or mixing formulas, errors still may be introduced due to insufficient sample sizes or possible systematic errors made in the measurement. Furthermore, it is difficult to provide a broad and sufficient experimental data space to fully evaluate a theoretical method. It is, of course, also useful to analyze similar problems through alternate approaches so as to provide a measure of validity to those measurements. 
An alternative to the physical measurement process is to perform Monte Carlo numerical simulations where, through the use of computers, physical variations can be eliminated. Such a numerical method requires the use of a packing algorithm to simulate particle arrangements in a medium and an electromagnetics code such as the method of moments (MoM) (or the T-matrix method) to solve for the fields within the medium under study. These methods are capable of accounting for all multiple scattering terms within the medium and, therefore, can be considered an exact solution for the problem. One such method uses a truncated $T$-matrix approach to estimate the extinction from a collection of up to 4000 spheres confined in a cubic volume [2], [14]. Extinction for the nonabsorptive scatterers is calculated by integrating the incoherent power scattered by the cubic volume over all observation angles (i.e., the amount of coherent power attenuated due to scattering). In effect, this method calculates the imaginary component of the effective permittivity due to multiple scattering. Difficulties with this method may arise, however, due to the fact that the volume is isolated in free space, thereby enhancing the contrast of the scattering volume being analyzed from its surroundings; ideally, the volume under study would be immersed in a homogeneous medium with a permittivity equal to that of the effective permittivity of the volume. Furthermore, because of this contrast problem it is likely that the calculated extinction would be dependent on the shape and size of the boundary walls, an effect not thoroughly explored in the paper.

In this light, we present a new method of determining the complex effective permittivity for two-dimensional (2D) dense random media. The core idea of the method is to confine a random distribution of particles within an imaginary boundary from which the coherent field may be determined over many realizations. Using a homogeneous material whose dimensions are the same as the imaginary boundary used for the random distribution of particles, we adjust the permittivity of the material so that the homogeneous medium coherent field is identical to the random medium coherent field. Thus, rather than ignoring the effect of the boundary shape and size, it is directly taken into account.

The 2-D problem is rigorously implemented here to provide insight and guidance for future work in analyzing the more general problem in three dimensions. This is accomplished by exploring the convergence effect as it depends on boundary shape and size and particle size. The 2-D problem does have direct application in modeling field interaction with fibrous material such as muscle tissue or dense prairie grasses. Results from this study can also provide a very important contribution to the study of dense media; that is, an "exact" solution for the effective permittivity that may be used as a benchmark for testing theoretical formulations such as the Polder-Van Santen mixing formula (this paper) and the QCA [10].

\section{REVIEW OF THE INCOHERENT METHOD}

The coherent method for determining $\epsilon_{\mathrm{eff}}$ in this article can be contrasted against the incoherent method for determining the extinction coefficient $-\kappa_{e}-$ given in [2] and [14]. The

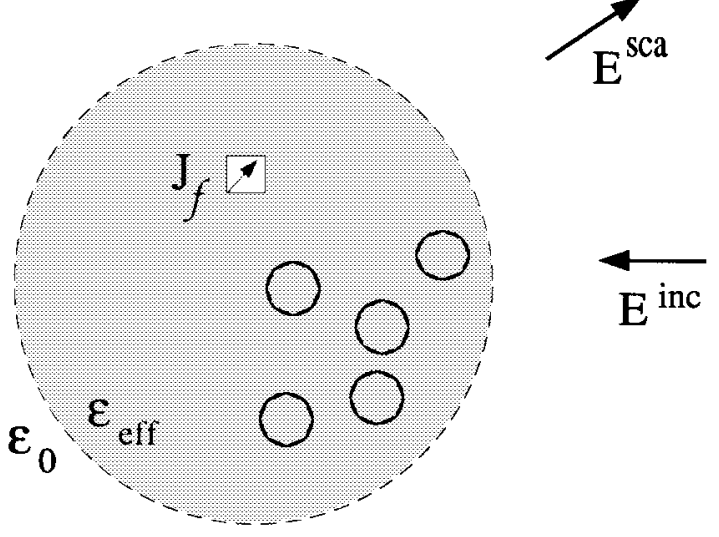

Fig. 1. Illustration of a volume current due to dielectric fluctuation in a random medium. Shown are some spherical components of the random medium.

purpose of this section is to make the distinction between the two methods clear, and to emphasize potential difficulties in the application of the incoherent method.

To begin, both methods examine the scattered field from a random collection of scatterers situated in free space (Fig. 1). For a given incident field, numerical calculations may be used to determine the scattered field, which is composed of a coherent and incoherent component (the coherent component does not vary between each realization $n$ )

$$
\overline{\mathbf{E}}_{n}^{\text {sca }}=\overline{\mathbf{E}}_{n}^{\text {incoh }}+\overline{\mathbf{E}}^{\text {coh }} \text {. }
$$

By performing many realizations $N$ of the random arrangement of scatterers, the coherent field may be found by averaging the scattered field

$$
\overline{\mathbf{E}}^{\text {coh }}=\frac{1}{N} \sum_{n=1}^{N} \overline{\mathbf{E}}_{n}^{\text {sca }} .
$$

The incoherent field for each realization may be found by solving (1). The coherent field is related to the size and shape of the confining boundary and arises from the random collection of scatterers behaving in part as a coherent structure with permittivity defined as $\epsilon_{\text {eff. }}$. The incoherent field arises from dielectric fluctuations between the inclusions and the background and it is this field which provides the basis for the incoherent technique.

Following the incoherent technique, the extinction coefficient is found by summing the power scattered into the incoherent field. This is accomplished via the integration

$$
\sigma_{N}=R \int_{0}^{2 \pi}\left\langle\left|\overline{\mathbf{E}}_{n}^{\text {incoh }}\right|^{2}\right\rangle d \phi_{s}
$$

where the $R$ factor normalizes the scattered power with respect to the distance from the origin. The extinction coefficient is determined by $\kappa_{e}=\sigma_{N} / A$, where $A$ is the area containing the random medium. Thus, the extinction coefficient is directly related to the incoherent field scattered from the random medium. Because the source and the observer are located outside of the random medium a potential problem with the incoherent technique arises; that is, the dielectric contrast between the random medium sample and that of free space 
has the potential of altering the behavior of fields from the way they would appear if the source and observer had existed in a medium whose permittivity was $\epsilon_{\text {eff }}$. Because the incoherent field is excited by the coherent field, the existence of a dielectric boundary on the incoherent fields would fundamentally alter the problem. The coherent method presented in this chapter circumvents this difficulty by directly taking into account the effective boundary of the random medium sample. To highlight this issue, a theoretical approach can be taken.

Fluctuations in the scattered field between different particle arrangement realizations are attributed to spatial variations between the permittivity of the inclusions/spaces $\epsilon(x, y, z)$ and that of the background $\epsilon_{\mathrm{eff}}$. These fluctuations act as a volumetric current distribution $\overline{\mathbf{J}}_{f}$ that can be concisely described by the wave equation as

$$
\nabla \times \nabla \times \overline{\mathbf{E}}_{1}-\kappa_{\mathrm{eff}}^{2} \overline{\mathbf{E}}_{1}=\omega^{2} \mu\left(\epsilon-\epsilon_{\mathrm{eff}}\right) \overline{\mathbf{E}}_{1}=i \omega \mu \overline{\mathbf{J}}_{f}
$$

where $\overline{\mathbf{E}}_{1}$ is the electric field within the boundary containing the random medium. Here we may pose two questions: 1) what is the contribution of a volume current $\overline{\mathbf{J}}_{f}$ to the coherent field and 2) what is the contribution of a volume current $\overline{\mathbf{J}}_{f}$ to the incoherent field? The answer to the first question is straightforward. By definition, the dielectric fluctuations do not contribute to the coherent field. We address the second question by relating the scattered field to the current fluctuation via the dyadic Green's function, $\overline{\overline{\mathbf{G}}}$

$$
\overline{\mathbf{E}}^{\text {sca }}=\int_{V} \overline{\overline{\mathbf{G}}} \cdot \overline{\mathbf{J}}_{f} d V .
$$

Here, $\overline{\overline{\mathbf{G}}}$ is the dyadic Green's function in the presence of a homogeneous dielectric scatterer with the prescribed boundary.

From the above discussion, it can be seen that the scattered field is a function of both the effective permittivity and the shape of the boundary. In contrast, the incoherent method uses the free-space Green's function and does not take into account these factors (a sparse medium assumption). This assumption runs counter to the method's original premise that the random collection of scatterers behave in part as a coherent structure with permittivity $\epsilon_{\mathrm{eff}}$.

To demonstrate that the conservation of energy approach that the incoherent method relies upon is not appropriate for inclusions that demonstrate absorption losses, we refer to Fig. 2. This figure illustrates the normalized extinction coefficient, $\kappa_{e} / k$ calculated by the coherent method (-), the incoherent method $\left(--_{-}\right)$, and the Polder-VanSanten mixing formula $(\cdots)$ as a function of volume fraction for two different particle permittivities $-\epsilon_{i}=3.6+i 0.01(*)$ and $\epsilon_{i}=3.6+i 0.1(0)$. It is expected that the Polder-VanSanten mixing formula provides the lower limit for the extinction coefficient because it only accounts for losses due to absorption (inclusion diameter in this example is $\lambda_{i} / 10$ ).

For the lossy case (indicated by o's) the extinction coefficient calculated by the incoherent method becomes unphysically smaller than the extinction coefficient predicted by the mixing formula, and is even less than the extinction coefficient predicted by the incoherent method for the low-loss inclusions shown by $*$ 's. This clearly indicates that the incoherent method

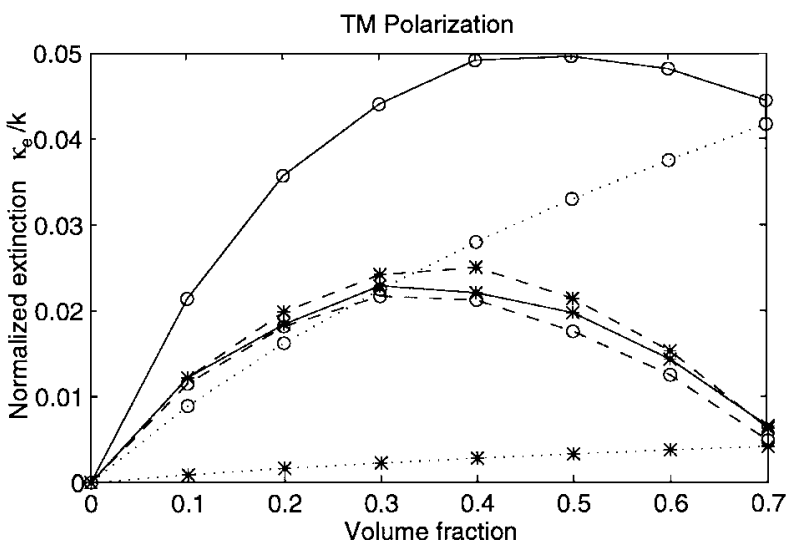

Fig. 2. Normalized extinction coefficient comparison between the coherent method (-), the incoherent method (- - -), and the Polder-Van Santen mixing formula $(\ldots)$ for two different inclusion permittivities: low-loss $\epsilon_{i}=3.6+i 0.01(*)$ and lossy $\epsilon_{i}=3.6+i 0.1(0)$.

is incapable of predicting the absorption loss in evaluating the extinction coefficient. It is interesting to note that by adding the absorption loss calculated by the Polder-Van Santen formula to the extinction calculated by the incoherent method, the result calculated by the coherent method can be reproduced approximately. However, this is not the case for the lowloss example in Fig. 2. In this case, the mean-field trapped within the boundary bounces many times which gives rise to an overestimation of extinction.

On a final, less critical note, it should be remembered that the extinction coefficient is related to the imaginary component of effective permittivity via the equation

$$
\kappa_{e}=2 k_{0} \operatorname{Im}\left(\sqrt{\epsilon_{\mathrm{eff}}}\right) .
$$

Calculation of $\operatorname{Im}\left(\epsilon_{\mathrm{eff}}\right)$ from $\kappa_{e}$ requires a knowledge of $\operatorname{Re}\left(\epsilon_{\text {eff }}\right)$, a quantity not provided by the incoherent method. The real part of effective permittivity is dependent on multiple scattering between particles as a measure of the mean-free path (i.e., the phase delay) and is an important parameter in a number of remote-sensing problems.

The coherent method described in this paper circumvents the problems discussed above by taking the effect of the boundary into account. In doing so, we are able to directly calculate both the real and imaginary components of effective permittivity. The validity of the coherent technique is emphasized in later sections where we show that the average field does, in fact, represent a coherent field scattered from a homogeneous body with permittivity $\epsilon_{\mathrm{eff}}$.

\section{Formulation OF THE COHERENT METHOD}

In this section, the numerical procedure for characterizing the effective permittivity of a random medium containing discrete scatterers is outlined. The procedure for determining the effective permittivity is a four step process. The first step is to generate a collection of particles with a specified volume fraction and particle arrangement contained within an imaginary boundary. Second, given an incident field, the MoM [5], [6] is then used to solve for the scattered field. Third, scattered fields for each realization of the collection of 


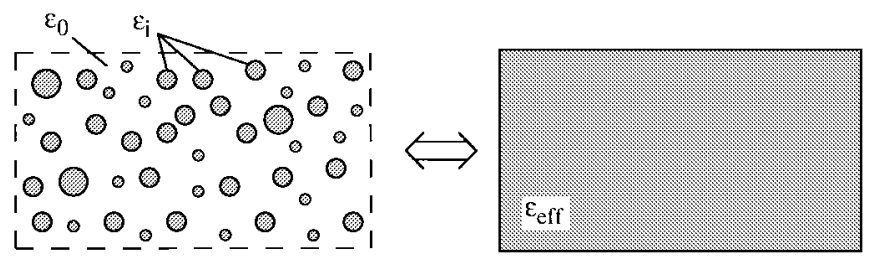

Fig. 3. Model for determining the effective permittivity for a random collection of scatterers.

particles are averaged over all observation angles to determine the coherent scattered field which is related to the shape and size of the imaginary boundary, as well as the effective permittivity. Finally, the effective permittivity of the medium is then found by finding the best fit between the coherent field from the Monte Carlo simulations and the field scattered from a homogeneous medium with permittivity $-\epsilon_{\mathrm{eff}}$ having the same boundary as the imaginary boundary. This step is an inverse scattering problem for which a novel technique based on the eigen-analysis in conjunction with the MoM is developed.

The packing algorithm used here [9] simulates the arrangements of particles with arbitrary shape, size, and orientation for "natural" dense media. From this arrangement of particles, a MoM solution for the scattered fields is obtained and the process is repeated many times to generate the statistics of the scattered field. This Monte Carlo simulation determines the average (coherent) scattered field as a function of observation angle $\theta$ for a random dense medium $\left\langle\overline{\mathbf{E}}_{\text {random }}^{s}(\theta)\right\rangle$. At this stage it is possible to retrieve the incoherent scattered power, which can be used directly to provide insight into the phase function used by radiative transfer. By taking the coherent scattered field one step farther, it is possible to determine the effective propagation constant, which can, in turn, be used to compare with QCA or even to create an empirical mixing formula for the simulated media.

To characterize the propagation constant of the mean field, we search for the dielectric constant of a uniform, homogeneous dielectric whose shape and size are the same as that used to bind the collection of random scatterers and which exhibits the same scattering pattern. It has been found that a unique effective permittivity does exist such that the scattering patterns from both the homogeneous and random media are very closely matched and, thus, the basic concept of the approach is validated. In the first case, a rectangular dielectric slab is used (Fig. 3). The MoM is used to determine the scattered field from this uniform dielectric as a function of observation angle and dielectric constant $\overline{\mathbf{E}}_{\text {uniform }}^{s}(\epsilon, \theta)$. A minimization technique can then be used to find the best fit permittivity for the observed scattered field of the uniform slab to the average field from the random scatterers

$$
\epsilon_{\mathrm{eff}}=\min _{\epsilon}\left[\left|\overline{\mathbf{E}}_{\text {uniform }}^{s}(\epsilon, \theta)-\left\langle\overline{\mathbf{E}}_{\text {random }}^{s}(\theta)\right\rangle\right|\right] .
$$

To find the minimum of (7) by iteration requires calculation of the volume currents and the scattered field for each new trial. While performing these calculations for each trial entails a straightforward electromagnetic numerical solution, since the dimensions of the boundary may be electrically large (as required by convergence criteria), the procedure can become very time consuming. However, a novel inversion solution is introduced in which using the MoM, the problem may be solved essentially only once-additional trials entailing a simple matrix multiplication.

\section{DIELECTRIC INVERSION MOM: EIGEN-ANALYSIS PROCEDURE}

The procedure of determining the scattered field from a dielectric body with inhomogeneous dielectric profile is a simple extension of the MoM. In this procedure, the dielectric dependence of the impedance matrix is explicitly separated from the equation, where it is highlighted that only the diagonal elements of the impedance matrix depend on the permittivity. By computing the eigenvectors and eigenvalues of the impedance matrix, it is possible to perform an inversion of the matrix prior to the inclusion of the permittivity term. Thus, calculation of the scattered field requires only a multiplication of the inverted eigenvector matrix and the dielectric term.

Consider a 2-D dielectric body illuminated by a plane wave. The incident wave induces a polarization current that becomes the source of the scattered field. The induced current is proportional to the total electric field inside the scatterer and is given by

$$
\overline{\mathbf{J}}=-i k_{0} Y_{0}(\epsilon-1)\left(\overline{\mathbf{E}}^{s}+\overline{\mathbf{E}}^{i}\right)
$$

where $\overline{\mathbf{E}}^{i}$ represents the incident wave and $\overline{\mathbf{E}}^{s}$ is the scattered field given by

$$
\overline{\mathbf{E}}^{s}=\int_{s} \overline{\mathbf{J}}\left(x^{\prime}, y^{\prime}\right) \cdot \overline{\bar{G}}\left(x, y ; x^{\prime}, y^{\prime}\right) d x^{\prime} d y^{\prime} .
$$

Here, $\overline{\overline{\mathbf{G}}}\left(x, y ; x^{\prime}, y^{\prime}\right)$ is the dyadic Green's function for 2D problems and $k_{0}$ and $Y_{0}$ are, respectively, the free-space propagation constant and characteristic admittance. Substitution of (9) in (8) results in the desired integral equation for the polarization current.

Solution to this integral equation must be obtained numerically. Using the MoM, the integral equation is cast into a matrix equation of the following form:

$$
\overline{\overline{\mathbf{Z}}} \overline{\mathbf{J}}=\overline{\mathbf{E}}
$$

where $\overline{\overline{\mathbf{Z}}}$ is the impedance matrix, $\overline{\mathbf{J}}$ is the polarization current, and $\overline{\mathbf{E}}$ is the excitation vector. The polarization current can be obtained by inverting (10) and is given by

$$
\overline{\mathbf{J}}=\overline{\overline{\mathbf{Z}}}^{-1}(\epsilon) \cdot \overline{\mathbf{E}}
$$

where $\overline{\bar{Z}}^{-1}$ is an implicit function of $\epsilon$. The minimization algorithm (depending on the initial guess) usually requires the calculation of $\overline{\mathbf{Z}}^{-1}$ many times, a numerical procedure which makes the inversion algorithm numerically very inefficient for large scatterers.

In what follows, a procedure for the calculation of the polarization current is presented that does not require repetitive evaluation of $\overline{\bar{Z}}^{-1}$ for different values of $\epsilon$. The minimization routine can be made efficient by noticing that the permittivity appears only in the diagonal elements of the impedance matrix. 
By splitting the impedance matrix into a diagonal term $\beta(\epsilon) \overline{\overline{\mathbf{I}}}$ and a modified impedance matrix term $\overline{\overline{\mathbf{W}}}$, we have

$$
\overline{\overline{\mathbf{Z}}}=\overline{\overline{\mathbf{W}}}+\beta(\epsilon) \overline{\overline{\mathbf{I}}}
$$

where $\overline{\overline{\mathbf{I}}}$ is the identity matrix and $\beta=1 /(\epsilon-1)$. Note that the $\overline{\bar{W}}$ matrix is dependent only on the scatterer geometry and is not dependent on the permittivity of the scatterer. By computing the eigenvalues $\overline{\bar{\Lambda}}$ and the eigenvector matrix $\overline{\overline{\mathbf{Q}}}$ of the $\overline{\bar{W}}$ matrix, it is known that

$$
\overline{\overline{\mathrm{W}}}=\overline{\overline{\mathrm{Q}}} \overline{\bar{\Lambda}} \overline{\mathrm{Q}}^{-1}
$$

Also noting that the identity matrix can be expressed by

$$
\overline{\overline{\mathbf{I}}}=\overline{\overline{\mathbf{Q}}} \overline{\overline{\mathbf{Q}}} \overline{\bar{Q}}^{-1}
$$

(11) can be written as

$$
\overline{\overline{\mathbf{J}}}=\overline{\overline{\mathbf{Q}}}[\overline{\overline{\mathbf{\Lambda}}}+\beta \overline{\overline{\mathbf{I}}}]^{-1} \overline{\mathbf{Q}}^{-1} \overline{\mathbf{E}}
$$

where the dependency of the polarization current on the permittivity has been made explicit. The calculation of the eigenvalues and eigenvector matrix is performed only once for a particular scattering geometry. The determination of the scattered field as a function of dielectric constant has then been converted from one of matrix inversion to matrix multiplication. Convergence of the minimization takes place after approximately $20 \mathrm{~s}$ (15 iterations) on a Sun 10 workstation for a $3 \lambda_{i} \times 1 \lambda_{i}$ dielectric slab where $\lambda_{i}$ is the wavelength in a dielectric slab with permittivity $\epsilon_{i}$. Equivalently, this is 300 unknowns for the TM polarization and 600 unknowns for TE polarization. The speed-up factor encountered is roughly two orders of magnitude.

\section{2-D MIXING FORMULA}

It is important to compare the numerical results from this analysis to theoretical models. At low frequencies, theories that relate the effective permittivity of a mixture to the permittivity of its components are termed mixing formulas, the most common of which is given by Polder and Van Santen [4]. The derivation given here parallels this work as it is applied to the 2-D problem.

We begin by examining the interaction of one particle of a mixture with the surrounding mean field $\langle\overline{\mathbf{E}}\rangle$. We wish to find the effective permittivity $\epsilon_{\mathrm{eff}}$, which is related to $\langle\overline{\mathbf{E}}\rangle$ by

$$
\langle\overline{\mathbf{D}}\rangle=\epsilon_{\mathrm{eff}}\langle\overline{\mathbf{E}}\rangle .
$$

The electric flux density $\bar{D}$ in the medium may be written as

$$
A\langle\overline{\mathbf{D}}\rangle=\int_{A_{h}} \epsilon_{h} \overline{\mathbf{E}} d s+\sum_{j} \int_{A_{j}} \epsilon_{i} \overline{\mathbf{E}} d s
$$

where $A$ is the area, $\epsilon_{h}$ and $\epsilon_{i}$ are the permittivity of the host and included materials, and the summation is taken over all of the particles $p_{j}$ in the medium. After some manipulation, we arrive at

$$
\epsilon_{\mathrm{eff}}\langle\overline{\mathbf{E}}\rangle=\epsilon_{h}\langle\overline{\mathbf{E}}\rangle+\sum_{j} f_{j} \frac{1}{A_{j}} \int_{A_{j}} \overline{\mathbf{E}}_{j} d s
$$

with the volume fraction $f_{j}=A_{j} / A$. If we assume that the mean-induced field in the particles may be expressed as the scalar product between a mean-normalized polarizability tensor $\left(\epsilon_{i}-\epsilon_{h}\right)\langle\bar{\alpha}\rangle$ and the mean field in the medium, we find that the summation in (18) may be written as

$$
\sum_{j} f_{j} \frac{1}{A_{j}} \int_{A_{j}} \overline{\mathbf{E}} d s=f\langle\overline{\bar{\alpha}}\rangle \cdot\langle\overline{\mathbf{E}}\rangle .
$$

This substitution would be correct for a low-frequency tenuous medium where individual particles do not perturb the field appreciably. The mean-polarizability tensor in two dimensions is split into the TM and TE polarization. For the TM polarization, this tensor is unity, independent of particle shape and size. For TE polarization, the polarizability tensor can easily be obtained for arbitrary particle shapes, as outlined by Sarabandi and Senior [7]. For a circular cylinder, the normalized polarizability tensor can be obtained analytically, and is given by

$$
\overline{\bar{\alpha}}=\left(\epsilon_{i}-\epsilon_{h}\right)\left[\begin{array}{cc}
\frac{2}{\epsilon_{i}+\epsilon_{h}} & 0 \\
0 & \frac{2}{\epsilon_{i}+\epsilon_{h}}
\end{array}\right] .
$$

For radially symmetric particles or assuming random orientation, we find the average polarizability as the average of the diagonal terms of the polarizability tensor. Thus, for the two polarizations we arrive at the mixing formulas for circular cylinders as

$$
\begin{aligned}
& \epsilon_{\mathrm{eff}}=\epsilon_{h}+f\left(\epsilon_{i}-\epsilon_{h}\right) \\
& \epsilon_{\mathrm{eff}}=\epsilon_{h}+f\left(\epsilon_{i}-\epsilon_{h}\right) \frac{2}{\epsilon_{i}+\epsilon_{h}}
\end{aligned}
$$

for the TM and TE polarizations, respectively.

\section{RESUlts FOR A Simple Boundary Shape}

Multiple trials of the presented method have been shown to provide values of effective permittivity independent of the boundary shape and independent of the boundary size once the size has passed some critical limit. This section describes the analysis of one such shape for varying particle size. The following section will address more completely the problem of algorithm convergence and shape independence.

The shape considered in this example is a rectangular $3 \lambda_{i} \times 1 \lambda_{i}$ dielectric slab where $\lambda_{i}$ is the wavelength in a dielectric slab with permittivity $\epsilon_{i}=3.6+i 1.0$ which reflects the real part of the inclusion permittivities. The size is chosen in this example so that at a volume fraction of $100 \%$, the discretization of the homogeneous slab of ten samples per wavelength will still be valid. The mean particle diameter is chosen to be one of $\lambda_{i} / 10,2 \lambda_{i} / 10$ or $3 \lambda_{i} / 10$, and follows 
a normal distribution with unity mean and $25 \%$ standard deviation $[N(1,0.25)]$. A minimum of 300 realizations were performed for each volume fraction and the mean-scattered field determined. From this mean-scattered field, the forward and backscatter directions were used as sample points in the minimization algorithm given by (7). Other points might have been used, but empirical testing showed that this was not necessary, convergence to a unique solution occurred for all volume fractions and all particles sizes used for both TM and TE polarizations of the incident and scattered fields.

Demonstration of the ability of the method to find a true effective permittivity is given in Figs. 4 and 5 for volume fractions of $10 \%$ and $80 \%$, respectively. It can be seen in both extreme cases that the bistatic scattered field from the homogeneous dielectric gives an excellent fit to the entire average bistatic scattered field from the random medium, even though only two points in the forward and backscatter direction are used in determining the effective permittivity. This is particularly remarkable in Fig. 4 (10\% volume fraction) where the locations of the scatterers for any particular realization is not reflective of the shape and size of the imaginary boundary. Furthermore, it has been observed that the solution to (7) is unique. This observation was made by plotting the error function in (7) over the space of realistic $\epsilon_{\mathrm{eff}}$ (i.e., $1.0 \leq$ $\epsilon_{\text {eff }}^{\prime} \leq \epsilon_{\text {inclusion }}^{\prime}$ and $0.0 \leq \epsilon_{\text {eff }}^{\prime \prime} \leq 10 \epsilon_{\text {inclusion }}^{\prime \prime}$ ), whereby it was noticed that only one local and global minimum existed.

Results from the simulations with varying volume fraction and particle size can be compiled onto a single plot that details the dependence of effective permittivity (real and imaginary components) on these parameters. Comparison can then be made with the theoretically derived mixing formula of Polder and Van Santen, given in the previous section. This comparison is shown in Figs. 6 and 7 for the TM and TE polarizations.

For the TM polarization it is interesting to note the exact agreement with the mixing formula model for small particle size for real permittivity. This behavior indicates that the mean-field approach taken by the mixing formula is indeed effective for small particles illuminated by an $E$-polarized field. As particle size increases, we note a deviation from the mixing-formula model. The imaginary component of the effective permittivity is especially illuminating as we note a measured loss term greater than the one predicted by the mixing formula, a result that indicates that the larger particles are contributing to the scattering process, a factor not accounted for in the mixing-formula model.

For the TE polarization we see a significant deviation from the mixing-formula results. The deviation of the numerical model, however, is an improvement over the mixing formula in that it follows the expected trend toward the limiting case of unity volume fraction. Similar to the case of TM polarization, the loss term for the $H$-polarized field demonstrates increased scattering losses due to the larger particle sizes as is expected.

This section has demonstrated how the presented method can be used to determine the effective permittivity for a random medium. The behavior of the effective permittivity was shown to behave in a manner consistent with what would be expected for changing volume fraction and particle size. An important question is whether or not the permittivity calculated

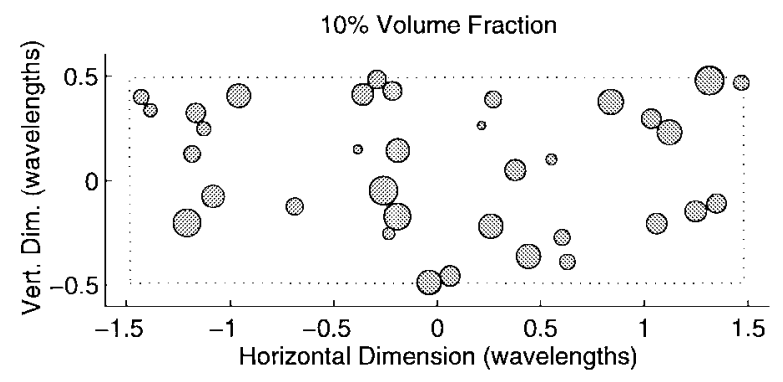

(a)

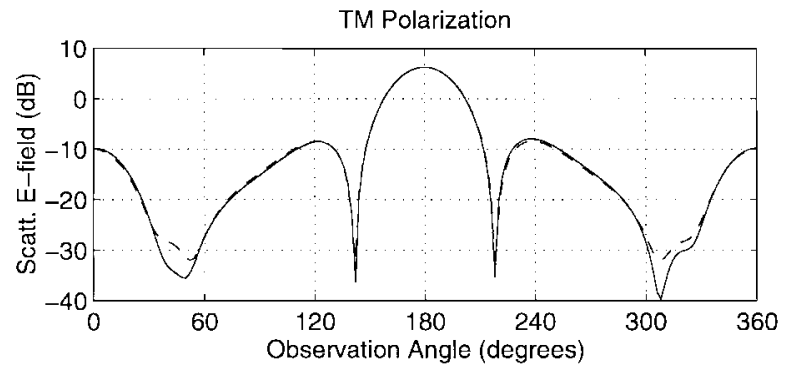

(b)

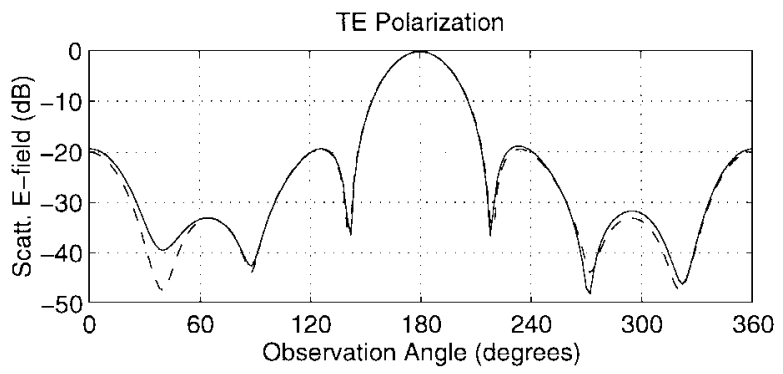

(c)

Fig. 4. Algorithm results for $10 \%$ volume fraction. (a) A sample collection of particles confined within an imaginary boundary. (b) Average scattered TM field (solid line) and the scattered field from a homogeneous dielectric with permittivity $\epsilon_{\text {eff }}$ (dashed line). (c) Average scattered TE field (solid line) and the scattered field from a homogeneous dielectric with permittivity $\epsilon_{\text {eff }}$ (dashed line). An average number of 34 particles were used for each realization. The incident field is broadside from the vertical direction.

is dependent on the shape and size of the bounding area used. The next section addresses this problem and offers answers as to how small the imaginary boundary may be to still reflect the large-scale behavior of the material parameters.

\section{CONVERGENCE CONSIDERATIONS}

This section seeks to offer evidence for the convergence of the solution for effective permittivity and to test independence of the method from the shape of the imaginary boundary used. To begin this controlled study, small particles of uniform diameter $\lambda_{i} / 10$ are used where, as before, $\lambda_{i}$ is the field wavelength within the included material whose permittivity is chosen to be $\epsilon_{i}=3.6+i 0.1$ - the background material being free space.

There are some important differences between the results shown in this section and those given in the previous section. In this section, the imaginary part of the scatterer permittivity is chosen to be ten times smaller than that used in the previous section to assure that multiple scattering is allowed to take 


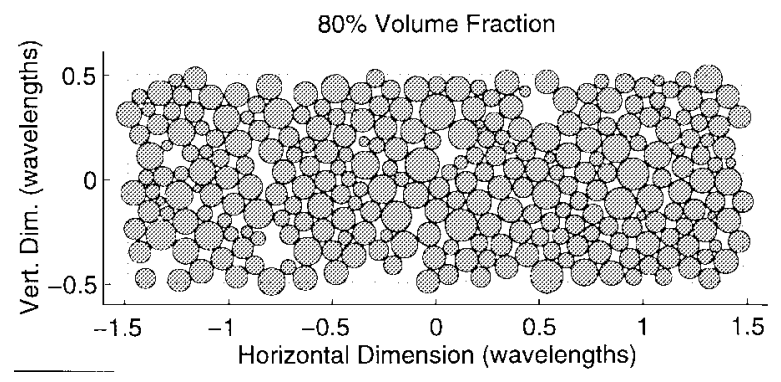

(a)

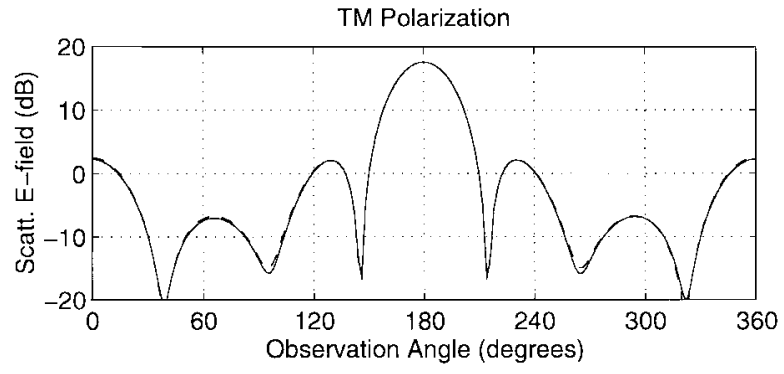

(b)

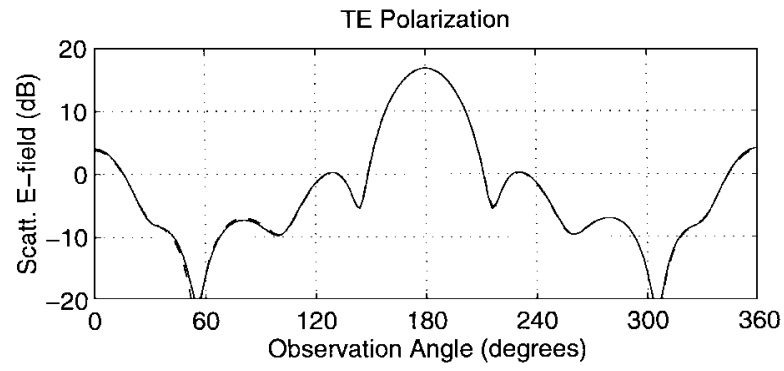

(c)

Fig. 5. Algorithm results for $80 \%$ volume fraction. (a) A sample collection of particles confined within an imaginary boundary. (b) Average scattered TM field (solid line) and the scattered field from a homogeneous dielectric with permittivity $\epsilon_{\text {eff }}$ (dashed line). (c) Average scattered TE field (solid line) and the scattered field from a homogeneous dielectric with permittivity $\epsilon_{\text {eff }}$ (dashed line). An average number of 278 particles were used for each realization.

place more significantly. As a consequence however, since the imaginary component of the effective permittivity is a factor of approximately 36 times less than the real component, numerical errors in the estimate of the imaginary component may be much more evident than those for the real component. Another difference of concern between this and the previous section is that scatterers of uniform diameter are used here. Thus, for high-volume fractions in the range of $50 \%$ to the limit of $91 \%$, the resulting collection of scatterer positions will be nearly crystalline and, therefore, anisotropic. To avoid this additional complexity, only volume fractions ranging from 10 to $50 \%$ are shown here.

We have already seen the ability of the method to achieve an excellent fit between the average scattered field from the random medium and the scattered field of a homogeneous dielectric. There are two questions that will be addressed in this section: 1) how large should the scattering boundary be and 2) how many realizations are required to determine the forward and backscatter field accurately? As it happens, the answer

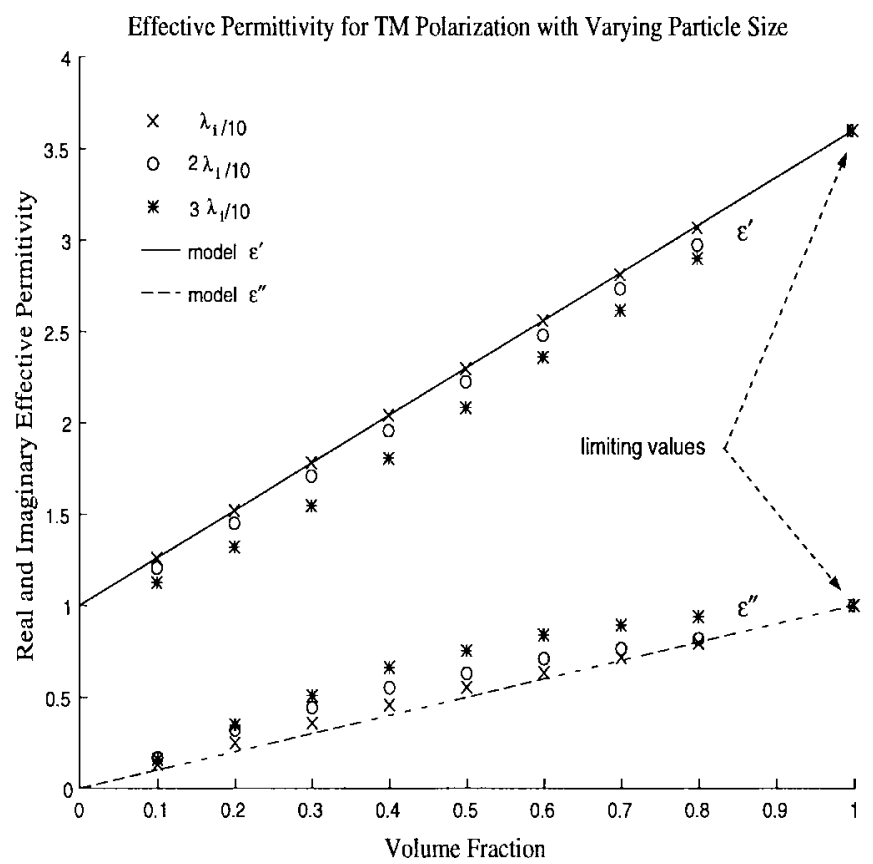

Fig. 6. Simulation and mixing formula results for TM polarization. Real (solid line) and imaginary (dashed line) components of the effective permittivity derived from the Polder-Van Santen mixing formula compared with effective permittivity obtained by numerical simulation. Symbols indicate different particle diameters of $\lambda_{i} / 10-\times, 2 \lambda_{i} / 10 \multimap$, and $3 \lambda_{i} / 10 \longrightarrow *$. Inclusion permittivity is $\epsilon_{i}=3.6+i 1.0$.

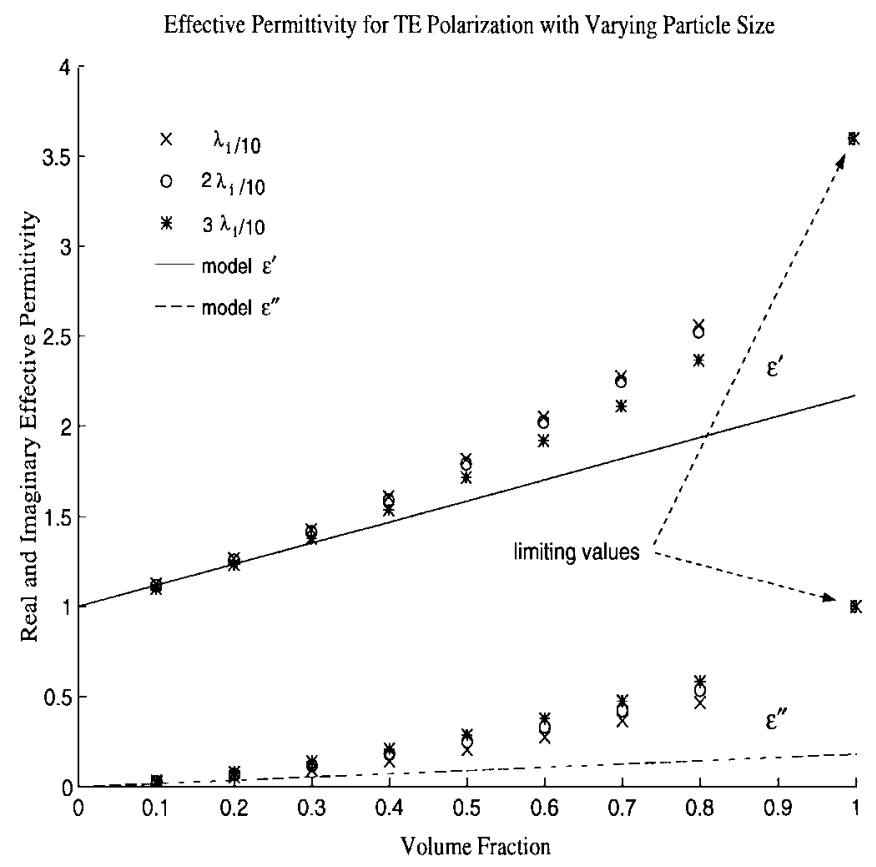

Fig. 7. Simulation and mixing formula results for TE polarization.

to these two questions are related; the larger the confining boundary gets, the larger is the variation of the electric field in the forward and backscatter directions. Thus, as the bounded area increases, so does the uncertainty of the calculated field.

To determine the convergence of the method for changing boundary size we refer to Fig. 8, which displays the real part 

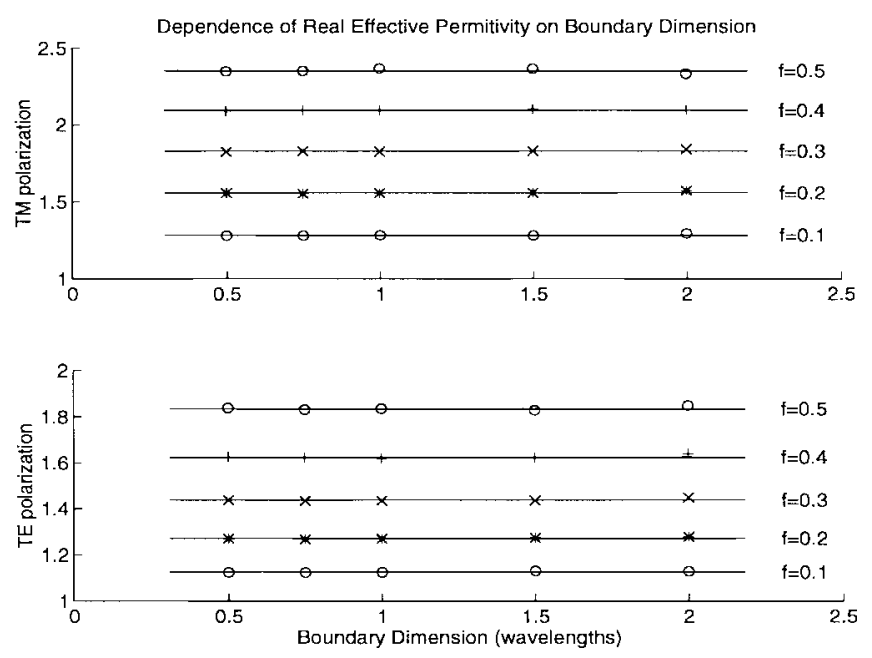

Fig. 8. Solution dependence on boundary dimension for TM and TE polarizations. Different lines on the graph represent the average permittivity for a given volume fraction of $f=0.1,0.2,0.3,0.4$ or 0.5 .
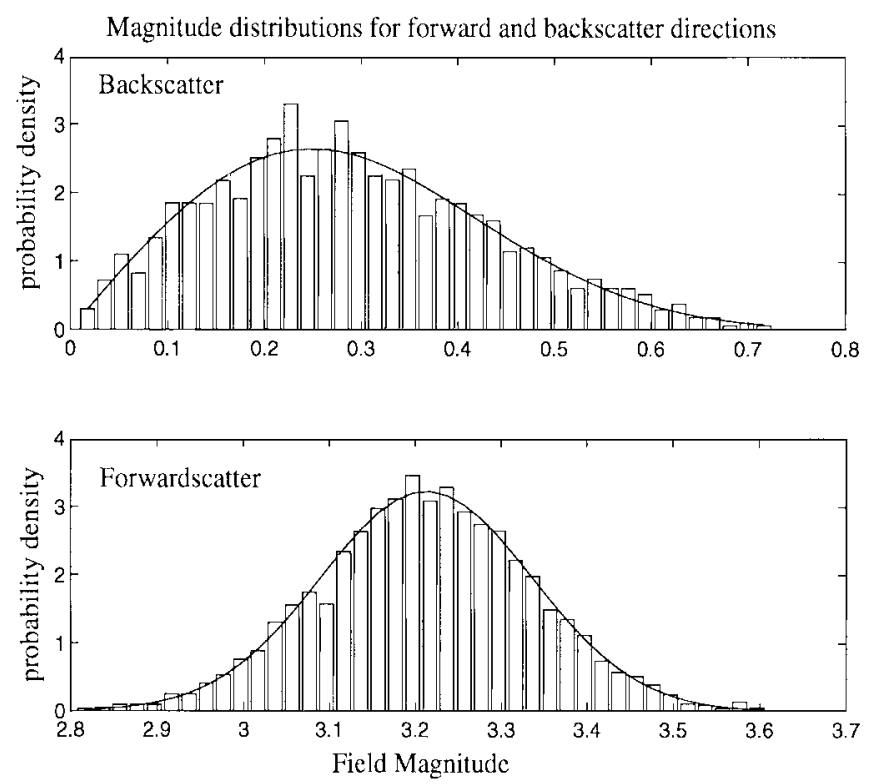

Fig. 9. Forward and backscatter field magnitude distributions. Numerical simulations (bar graph) follow the Rice-Nakagami distribution (solid line). This example is for a square box of dimension $1.5 \lambda \times 1.5 \lambda, 40 \%$ volume fraction TE polarization.

of the effective permittivity for the TM and TE polarizations as a function of boundary size for differing volume fractions of particles. We note in the figure that the solution is stable for a wide range of boundary sizes (i.e., horizontal lines). As boundary size decreases farther from the displayed range, it is expected that errors will increase due to an insufficient number of scatterers located within the boundary. At the other extreme of a large boundary size, it is expected that the numbers will become error prone due to the increased uncertainty in measuring the forward and backscattered field.

Individual realizations of the forward and backscatter field magnitude accurately follow a Rice-Nakagami distribution (Fig. 9) [11, p. 94] where the mean value and standard
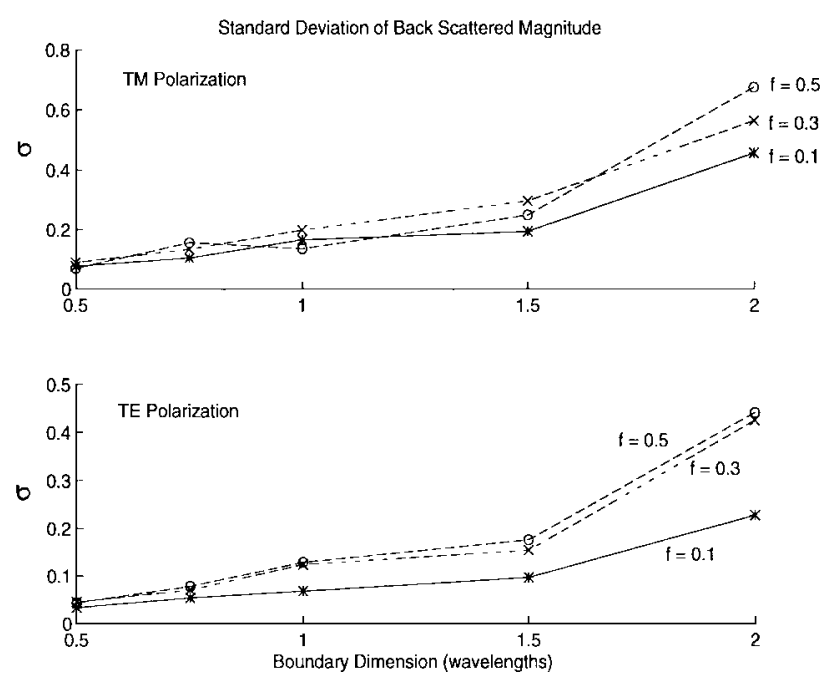

Fig. 10. Standard deviation of backscattered field magnitude for three different volume fractions $(0.1,0.3$, and 0.5$)$ and both TM and TE polarizations. Similar behavior was observed for the forward-scattered field as well.

deviation are dependent on the boundary shape and size. To explore the size dependence of the standard deviation, Fig. 10 shows the standard deviation of backscatter magnitude for changing boundary size and volume fraction for both the TM and TE polarization. It can be seen from the graphs that the variation in observed field is not strongly dependent on volume fraction, but does increase steadily as the boundary size increases. If we make the observation that the standard deviation increases by a factor of two between the boundary sizes of $1.5 \lambda_{i}$ and $2.0 \lambda_{i}$, to achieve a given accuracy of field magnitude, the larger boundary would require four times as many simulations. The ideal boundary size would then appear to fall somewhere between $1.0 \lambda_{i}$ and $1.5 \lambda_{i}$.

There are three basic geometries that have been tested: a circular disk, a rectangular slab, and a square. Real and imaginary effective permittivities for these shapes are shown in Fig. 11 (note that the imaginary component is multiplied by a factor of ten) as a function of volume fraction for both TM and TE polarizations. Also given for comparison are the mixing formula results obtained from (15) and (16). From this figure it can be seen that there is essentially an exact match between the real permittivities for all three shapes with some small deviation being noticed for the imaginary part of the effective permittivity. These differences are likely due to difficulties in estimating an imaginary component that is much smaller than the real component of the permittivity with a relatively small number of realizations.

So far, in this section, it has been demonstrated that the proposed technique yields stable results independent of boundary shape and size. It was also shown that the forward and backscattered field magnitudes accurately follow a Rice-Nakagami distribution whose variance increases as a function of boundary dimension. One final test is to show the dependence of minimum boundary size on particle size. As particle size increases, it is expected that increased scattering and reduction in the number scatterers for a given volume fraction will increase the minimum boundary dimension for 

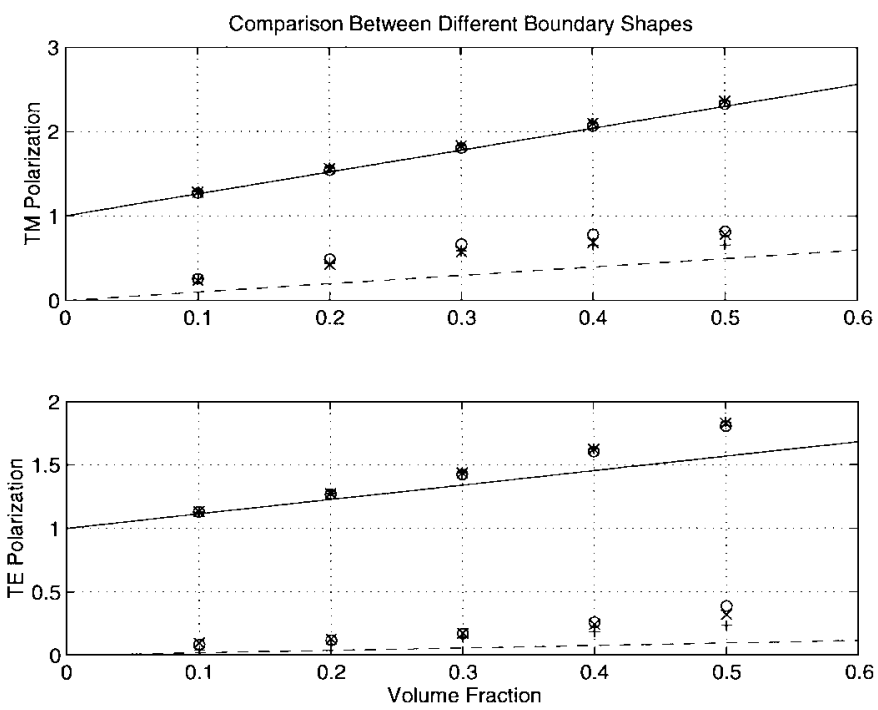

Fig. 11. Effective permittivity comparison for differing boundary shapes Shown are the real and imaginary permittivities for three different boundary shapes: o-circular disk (diameter $\left.=2 \lambda_{i}\right),+$-rectangular slab $\left(3 \lambda_{i} \times \lambda_{i}\right)$ and $\times$-square box $\left(1.5 \lambda_{i} \times 1.5 \lambda_{i}\right)$. The imaginary part of the effective permittivity is multiplied by ten so that both parts of the effective permittivity may be displayed on the same graph. Lines indicate results from the 2-D Polder-Van Santen mixing formula.
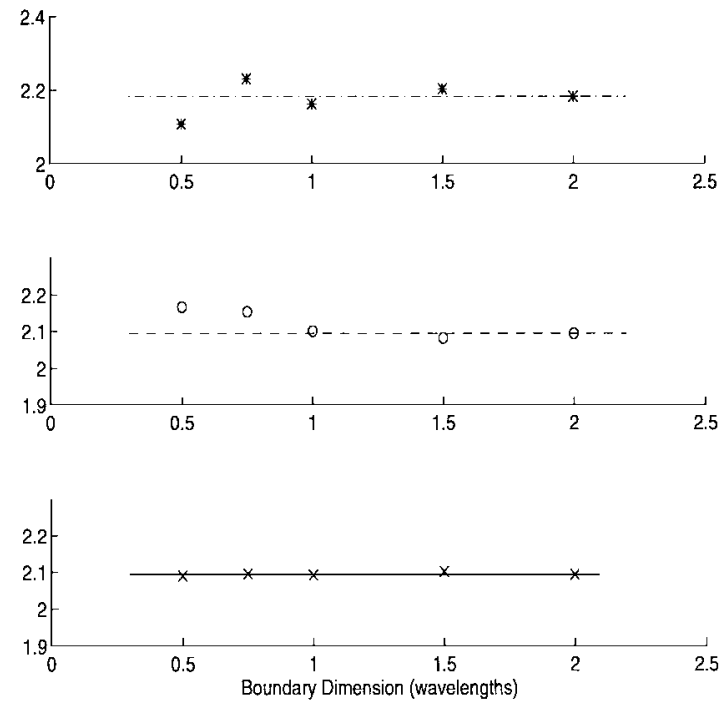

Fig. 12. Real part of effective permittivity versus boundary dimension for three different particle sizes: $\lambda_{i} / 10-\times, 2 \lambda_{i} / 10-0,3 \lambda_{i} / 10 \longrightarrow *$ and a volume fraction of $40 \%$ TM polarization.

which the algorithm will converge. Fig. 11 demonstrates this behavior by plotting the effective permittivity as a function of boundary dimension for three different particle sizes $\left(\lambda_{i} / 10,2 \lambda_{i} / 10\right.$, and $\left.3 \lambda_{i} / 10\right)$ at a volume fraction of $40 \%$ for a TM polarized field. From this figure it is evident that the $\lambda_{i} / 10$ particles converge to a stable value even at the smallest of boundary sizes used. As particle size increases, so does the required boundary size. Particles of diameter $3 \lambda_{i} / 10$ require a boundary equal to or larger than two wavelengths before reaching convergence. Thus, care must be taken to insure that: 1) boundary size is large enough to insure convergence and
2) a sufficient number of realizations is used to sufficiently calculate the coherent field.

\section{CONCLUSION}

This paper has put forward a new technique for numerically determining the effective permittivity of a random medium in two dimensions. A key component of the technique is a MoM/eigen-analysis inversion technique that efficiently calculates the volume currents for a deterministic body with variable permittivity. This inversion technique was applied to a range of random media that varied particle size, volume fraction, boundary size, and shape. Results from the inversion technique where compared with the 2-D Polder-Van Santen mixing formula and shown to agree in the small-particle lowvolume fraction limit. The last section then addressed the problem of boundary size, particle size, and convergence of the solution. The extensive study performed in this section of the paper will provide insight as how to approach the 3D problems. The strength of the method is to create multiple scattering numerical solutions for effective permittivity for a group of canonical situations. Such solutions can then be used to analyze theoretical methods such as the Polder-Van Santen mixing formula, the QCA, or future models that address the dense random media problem.

\section{REFERENCES}

[1] M. Lax, "Multiple scattering of waves-II: The effective field in dense systems," Phys. Rev., vol. 85, no. 4, pp. 621-629, Feb. 15, 1952.

[2] C. C. Lu, W. C. Chew, and L. Tsang, "The application of recursive aggregate T-matrix algorithm in the Monte Carlo simulations of the extinction rate of random distribution of particles," Radio Sci., vol. 30, no. 1, pp. 25-28, Jan./Feb. 1995.

[3] C. E. Mandt, Y. Kuga, L. Tsang, and A. Ishimaru, "Microwave propagation and scattering in a dense distribution of nontenuous spheres: Experiment and theory," Waves Random Media, vol. 2, pp. 225-234, 1992.

[4] D. Polder and J. H. Van Santen, "The effective permeability of mixtures of solids," Physica, vol. 12, no. 5, pp. 1257-1271, Aug. 1946.

[5] J. H. Richmond, "Scattering by a dielectric cylinder of arbitrary crosssection shape," IEEE Trans. Antennas Propagat., vol. AP-13, pp. 334-341, May 1965.

[6] _ , "TE-wave scattering by a dielectric cylinder of arbitrary crosssection shape," IEEE Trans. Antennas Propagat., vol. AP-14, pp. 460-464, July 1966.

[7] K. Sarabandi and T. B. A. Senior, "Low-frequency scattering from cylindrical structures at oblique incidence," IEEE Trans. Geosci. Remote Sensing, vol. 28, pp. 879-885, Sept. 1990.

[8] K. Sarabandi, "A technique for dielectric measurement of cylindrical objects in a rectangular waveguide," IEEE Trans. Instrum. Meas., vol. 43, pp. 793-798, Dec. 1994.

[9] P. R. Siqueira, K. Sarabandi, and F. T. Ulaby, "Numerical simulation of scatterer positions in a very dense medium with an application to the two-dimensional born approximation," Radio Sci., vol. 30, no. 5, pp. 1325-1339, Sept./Oct., 1995.

[10] P. R. Siqueira and K. Sarabandi, "Numerical evaluation of the twodimensional quasicrystalline approximation," IEEE Trans. Antennas Propagat., to be published.

[11] H. Stark and J. W. Woods, Probability, Random Processes, and Estimation Theory for Engineers. Englewood Cliffs, NJ: Prentice-Hall, 1986.

[12] C. T. Tai, Dyadic Green Functions in Electromagnetic Theory. New York: IEEE Press, 1994, Ser. Electromagn. Waves.

[13] L. Tsang, J. Kong, and R. Shin, Theory of Microwave Remote Sensing. New York: Wiley, 1985.

[14] L. Tsang, C. E. Mandt, and K. H. Ding, "Monte Carlo simulations of the extinction rate of dense media with randomly distributed dielectric spheres based on solution of Maxwell's equations," Opt. Lett., vol. 17, no. 5, pp. 314-316, Mar. 1992. 


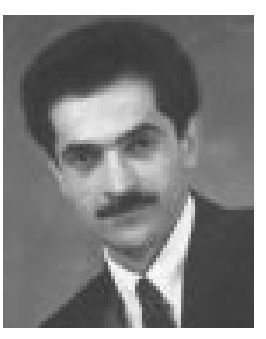

Kamal Sarabandi (S'87-M'90-SM'92) received the B.S. degree in electrical engineering from Sharif University of Technology, Tehran, Iran, in 1980, and the M.S.E. (electrical engineering), M.S. (mathematics) and Ph.D. (electrical engineering) degrees, in 1984, 1986, and 1989, respectively, from the University of Michigan, Ann Arbor.

From 1980 to 1984 he worked as a Microwave Engineer in the Telecommunication Research Center. He is presently an Associate Professor in the Department of Electrical Engineering and Computer Science at the University of Michigan, Ann Arbor. He has 15 years of experience with microwave sensors and radar systems. In the past seven years he has served as Principal Investigator and Co-Investigator on many projects sponsored by NASA, JPL, ARO, and ONR, all related in some way to microwave and millimeter-wave radar remote sensing. He has published four book chapters and more than 60 papers in refereed journals on electromagnetic scattering, random media modeling, microwave measurement techniques, radar calibration, application of neural networks in inverse scattering problems, and microwave sensors. He has also had more than 110 papers and presentations in national and international conferences and symposia on similar subjects

Dr. Sarabandi is listed in Who's Who in Electromagnetics and is the Chairman of the Geoscience and Remote Sensing Society Southeastern Michigan chapter. He was a recipient of a 1996 Teaching Excellence Award and the 1997 Henry Russel Award from the Regent of The University of Michigan.

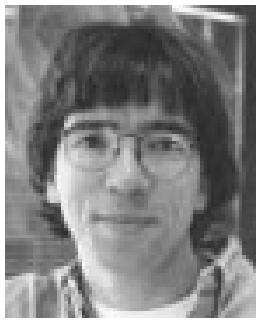

Paul R. Siqueira received the B.S. degree in electrical engineering from Iowa State University, Ames, and the Ph.D. in electrical engineering from the Radiation Laboratory, University of Michigan, Ann Arbor, in 1987 and 1996, respectively.

Prior to entering the University of Michigan, he worked at the University of Chicago, IL, applying image processing techniques to problems in astronomy and molecular biology. Currently, he is a Staff Scientist at the Radar Science and Engineering Section, Jet Propulsion Laboratory, Pasadena, CA, where he is investigating radar interaction and imagery of tropical rain forests. His interests are in the study of electromagnetic phenomena applied to problems in remote sensing. 